Onkologe 2004 · [Suppl 1]10:S5 DOI 10.1007/s00761-004-0668-5 C) Springer-Verlag 2004

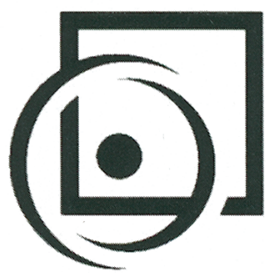

W. Budach · M. Bamberg · Radiologische Universitätsklinik Tübingen

\title{
Verantwortung übernehmen...
}

\section{Deutscher Krebskongress 2004}

v erantwortung übernehmen ist der Leitsatz des diesjährigen Deutschen Krebskongresses in Berlin. Ärztliche Verantwortung bedeutet auch und im besondern Maße die Verpflichtung zur Fortbildung. Der Deutsche Krebskongress bietet wie kein anderer deutschsprachiger Kongress Kollegen aller Fachdisziplinen ein umfassendes Fort- und Weiterbildungsprogramm zur Prävention, Behandlung und Nachsorge maligner Erkrankungen an. In Ergänzung dieses umfassenden Programms ist es dieses Jahr ist es erstmals gelungen, zusätzlich einen „EducationalBand“als Supplementheft $z u$,Der Onkologe" rechtzeitig zum Kongress fertig zustellen.

Die Autoren der ausgewählten Einzelbeiträge sind auch Referenten der jeweils zu Beginn der einzelnen Kongresstage stattfindenden Fortbildungsveranstaltungen. Bei der Wahl der Schwerpunkten wurden bewusst Themen aufgegriffen, die im "Educational-Band" des letzten Krebskongresses nicht berücksichtigt wurden und dennoch von großem Allgemeininteresse sind. Um der zunehmenden Interdisziplinarität der Behandlung von bösartigen Tumoren Rechnung zu tragen, werden wichtige Themenschwerpunkte, wie z.B. die Therapie des Magenkarzinom, aus dem Blickwinkel verschiedener Fachdisziplinen beleuchtet. Dabei werden sowohl die derzeitige Standardtherapie als auch aktuelle Entwicklungen dargestellt. Auch auf wichtige Erkenntnisse aus Grundlagenforschungwirdschlaglichtartigeingegan- gen und auf deren potentielle klinische Bedeutung hingewiesen.

Im Bestreben, die kontinuierliche und interdisziplinäre Fortbildung zu unterstützen, soll der vorliegende „Educational-Band" zum 26. Deutschen Krebskongress als aktiver Beitrag aufgefasst werden.

Tübingen im Februar 2004

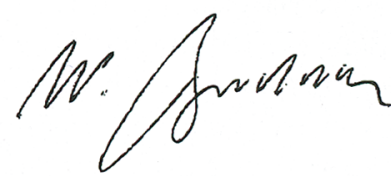

W. Budach

Kongresssekretär<smiles>CCCC</smiles>

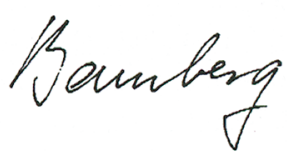

M. Bamberg Kongresspräsident

\section{Korrespondierender Autor Prof. Dr. W. Budach}

Radiologische Universitätsklinik Tübingen Hoppe Seyler Str. 3, 72076 Tübingen E-Mail:Wilfried.Budach@med.uni-tuebingen.de 